\title{
Spacetime functionalism in general relativity and quantum gravity
}

\author{
Karen Crowther ${ }^{1}$ - Niels S. Linnemann ${ }^{2} \cdot$ Christian Wüthrich $^{3}$
}

Published online: 16 June 2020

(c) The Author(s) 2020

Physics has seen decades of efforts to come to grips with the foundations of quantum physics and to articulate a quantum theory of gravity. Despite many promising ideas and occasional breakthroughs, progress has been slow. In recent years, more and more philosophers of physics have arrived at the conclusion that a key to progressing on at least some stubborn problems in the foundations of physics may be to reconceive the notion of spacetime in ways that transcend the basic substantivalist or relationalist positions. In the foundations of quantum mechanics, general relativity (GR), and quantum gravity, spacetime functionalism has emerged as a promising approach to the vexed issue of the nature of spacetime. In quantum mechanics and in GR, spacetime functionalism has opened new interpretative avenues, and in quantum gravity it is possibly essential for understanding how spacetime emerges from a fundamentally non-spatiotemporal structure. In light of these recent developments, this special issue on spacetime functionalism in GR and quantum gravity offers a timely collection of newly written articles advancing our growing appreciation of the promises-and the limitations - of spacetime functionalism.

The motivation for a functionalist approach in any area of philosophy is the recognition that many concepts of interest are multiply-realisable: functionalism holds that a given concept of interest is best understood by means of the role that concept plays, rather than by (any, or all, of) its specific instances or realisations. Functionalism is most familiar in the context of philosophy of mind, where it is the statement that what makes something a mental state of a certain type is determined by the way it

$\bowtie \quad$ Niels S. Linnemann

niels.linnemann@uni-bremen.de

Karen Crowther

karen.crowther@ifikk.uio.no

Christian Wüthrich

christian.wuthrich@unige.ch

1 Department of Philosophy, University of Oslo, Georg Morgenstiernes hus, Blindernveien 31, 0313 Oslo, Norway

2 Institute of Philosophy, University of Bremen, Enrique-Schmidt-Str. 7, 28344 Bremen, Germany

3 Department of Philosophy, University of Geneva, 5 rue de Candolle, 1211 Geneva 4, Switzerland 
functions - the role it plays - in the system of which it is a part. Motivating this view is the belief that a mental state is not primarily linked to the internal constitution of the creature which experiences it, but rather is multiply-realisable in different types of creatures with a rather different material constitution. The canonical example is the mental state of 'being in pain', which may be characterised as a state that tends to be caused by bodily injury or disease, to produce the belief that something is wrong with the body and the desire to be out of that state, and to typically cause wincing or wailing (Levin 2018). A functionalist perspective would say that all and only those creatures whose internal states meet these conditions, or play these roles, are in pain. In humans, this state might be realised by a particular type of neural activity, such as C-fibre stimulation - in which case, a human is in pain just in case they are undergoing C-fibre stimulation. The state of being in pain, however, could also be realised in different ways in creatures whose internal compositions are very different from those of human beings.

As the example shows, a functionalist approach to understanding a particular concept involves two steps. First, the concept must be defined in terms of its functional role-in other words, a functionalist must establish (or, at least, assert) that there is nothing more to the concept in question than the particular role that the functionalist specifies. In the example above, this involves asking 'What is the functional role of 'being in pain'?', which yields the characterisation of 'being a state that tends to be caused by bodily injury or disease, to produce the belief that there is something wrong with the body, etc.'. The second step involves then identifying, within the particular system of interest, the entity (structure, or process) that fulfils this role and to show how it does so.

Spacetime functionalism is the employment of functionalism to understand the concept of spacetime in the context of physical theories. As modern theories of physics have become increasingly sophisticated, it has become less obvious which structures in these theories should be taken as spacetime. Spacetime functionalism proceeds from the belief that spacetime is multiply-realisable by different structures, depending on the theory in which they feature. Spacetime functionalism states that what it is to be spacetime is nothing other than to fulfil a particular functional role. In identifying a particular structure as spacetime within a theory, the functionalist must follow the two-step procedure outlined above. First, they provide a definition of spacetime in terms of its functional role; second, they point out that this role is played by some entity in the theory. That entity which plays the functional role of spacetime in that theory, just is spacetime.

Spacetime functionalism has in recent years come into prominence in different areas of philosophy of physics. First, functionalism has been called to aid in the context of non-relativistic quantum mechanics by Wallace (2012) in his defence of an Everettian interpretation and by Albert (2013) in order to explain the emergence of space(time) and its content in wave function realism. Since time is supposed to be present at the fundamental level (together with the wave function), the concern in this area is really the emergence of three-dimensional physical space and three-dimensional objects in it from the wave function inhabiting a much higher-dimensional space$3 N$-dimensional configuration space. Here, $N$ is the total number of particles in the system described by the theory, ultimately the entire universe. Thus, there is a sense 
in which this is more of a 'space' functionalism, rather than spacetime functionalism. This is different in the second and third subfields of philosophy of physics in which spacetime functionalism has been deployed.

The second area is GR and related classical theories of spacetime, where the notion has been introduced by Knox $(2013,2014,2019) .{ }^{1}$ Spacetime functionalism in this domain has a direct precursor in the dynamical approach to GR articulated by Harvey Brown and Oliver Pooley (Brown 2005; Brown and Pooley 2006). Simply put, the dynamical approach demands an explanation of the 'chronogeometric significance' of the metric field, i.e., how rods and clocks measure the metric field. For Brown, this significance is acquired in dynamic coupling relations between the matter fields and the metric field, and does not inhere in the metric field itself. Thus, chronogeometric significance is a functional role that, first, can (at least partly) be attributed to various kind of fields, and that, second, is instantiated only if adequate dynamical coupling relations to other fields are in place. Given that chronogeometricity is usually seen as a core feature of what we mean by spacetime, the dynamical approach can thus naturally be conceived as a form of spacetime functionalism. Building on the dynamical approach, Knox's spacetime functionalism asserts that spacetime is whatever plays the role of inertial structure.

The third area is quantum gravity. Many approaches to quantum gravity seem to suggest that the fundamental structure of our world is much less spatiotemporal than the manifest world is, or as it is according to GR. If such non-spatiotemporality at the fundamental level is borne out, then it may render those theories empirically incoherent (Huggett and Wüthrich 2013) or non-physical (Lam and Esfeld 2013, §4.2). Incipient in their own response to the challenge of empirical incoherence in Huggett and Wüthrich (2013), Lam and Wüthrich (2018) have recently argued much more explicitly in their response that spacetime functionalism can bridge the gap between possibly non-spatiotemporal fundamental ontologies and the emerging spatiotemporality. In their view, it is sufficient to demonstrate how the functionally relevant aspects of relativistic spacetimes can be recovered from the fundamental structures in order to dispel concerns about the non-spatiotemporality of ontologies in quantum theories of gravity.

As the high-quality contributions at the international two-day conference on spacetime functionalism at the University of Geneva (1-2 March 2018) showed, the story does not end here. Spacetime functionalism has become an active field of research which has seen many important contributions recently. Thus, we sought to bring together much of this recent work in this special issue. As editors, we are thankful to all contributing authors: their work not only shows how much of an exciting research topic spacetime functionalism is, but in fact significantly advance it. Or so we believe-may the reader be the judge!

The papers fall into two main groups: spacetime functionalism in GR and related classical theories on the one hand, and in quantum gravity on the other. In the following, let us briefly introduce the contributions to the special issue.

\footnotetext{
1 The exact theoretical scope of this program is not clear, but it definitely involves general relativistic and general relativistic-like theories as well as various variations of Newtonian theories.
} 


\section{Brief overview of the contributions}

\section{Spacetime functionalism in GR}

The first paper of the special issue by James Read and Tushar Menon provides constructive criticism of Knox's version of spacetime fuctionalism. Read and Menon show how inertial structure and the operational meaning of spacetime can come apart in some models of GR and in some alternative classical spacetime theories. This divergence, they argue, is problematic for a programme motivated to closely track the operational significance of spacetime. They discuss various ways to modify or amend Knox's inertial-frame functionalism in order to circumvent the problem. They end with a discussion of the relation between inertial-frame functionalism and the wider dynamical approach and argue that these are largely orthogonal.

Jim Weatherall's contribution, a straight-on reckoning with two 'dogmas' behind the dynamical approach, is thus a welcome contribution to this special issue as it provides important clarification of widely held beliefs within the dynamical approach camp. Weatherall's paper, which he presents "in reaction to and sympathy with" a recent paper by Read (2020b), identifies the two dogmas as the ideas that, first, the stipulation of spacetime geometry has no implications for the behaviour of matter, and, second, the postulation of what is known as the 'strong equivalence principle' is enough to have matter be 'adapted' to spacetime geometry. Weatherall questions both of these ideas, and irenically concludes that careful consideration of the details of the relationship between dynamics and geometry dissolves much of the often advertised disagreement between the geometrical and the dynamical approaches.

In his article, David Baker argues that Knox's inertial-frame functionalism fails to capture the richness of our concept of spacetime and so lacks the generality one would expect from a spacetime functionalist framework. According to Baker, spacetime is a cluster concept: the criterion of playing the role of inertial structure can neither be necessary nor sufficient for calling it spacetime. Admittedly, Knox can easily bite the bullet that her or improved variants of her account will in the end just be valid for a certain sector of spacetime theories. Arguably more problematic, however, is Baker's follow-up that fundamentality can and is often traded as a central aspect of the spacetime concept-perhaps even of more importance than inertial structure-but is not at all touched by Knox's functionalism.

A different form of functionalism in GR concerns the status of the energymomentum tensor in GR: should it perhaps be given a functionalist reading as well, as suggested by Lam (2011) or Read (2020a)? Patrick Dürr's contribution pursues this question. For this, he appraises functionalist accounts of gravitational energies as opposed to, first and foremost, the eliminativist take on gravitational energy by Hoefer (2000); he arrives at the conclusion that the realist case for gravitational energy via a functionalist account will require substantive modification for it to prove fecund. 


\section{Spacetime functionalism in QG}

In their contribution, Vincent Lam and Christian Wüthrich follow up on their earlier work having first applied spacetime functionalism to quantum gravity. The main aim of their paper is to distinguish their form of spacetime functionalism in motivation, scope, and implications from spacetime functionalism as it has been advocated in the context of quantum mechanics and GR. Lam and Wüthrich argue that their spacetime functionalism, unlike that in other areas, does not compete with alternative interpretations of the relevant theories. Rather, it articulates and explicates the tasks of physical theorizing in quantum gravity. As such, they conclude, it is perfectly compatible with a realist take on quantum gravity, and even with a realist understanding of GR.

On their part, Michael Esfeld and Niels Linnemann are critical of the extent to which radical claims of spacetime emergence in quantum gravity are ultimately justified. In his contribution, Esfeld likens the current stage of quantum gravity research to the historical development of quantum mechanics and quantum fields throughout which claims had been abundant that the theories necessarily implied the dismissal of local 'beables' - concrete local things that we can straightforwardly take to be realand thus the conception of spacetime in any form. Just as the (putative) problem of the non-fundamentality of spacetime in quantum mechanics has been circumvented by Bohmian mechanics, Esfeld believes that the ontological problem so heavily problematised by philosophers of quantum gravity might evaporate after sufficient interpretational work.

Baptiste Le Bihan differentiates the problem of empirical coherence through identifying an epistemological and an ontological component whose difficulties are respectively compared to that of the easy and the hard problem of consciousness. Le Bihan's differentiation, however, continues: depending on which exact form of functionalism one adheres to, the hard problem is (dis)solved or not solved at all; only the easy problem is straightforwardly solved as suggested by Lam and Wüthrich (2018), or so Le Bihan argues.

Following largely Le Bihan in his distinction between the easy and the hard problem, Linnemann argues that the easy problem (the epistemological problem of empirical coherence) has been overstated by Huggett and Wüthrich (2013) from the start. Secondly, contra both Lam and Wüthrich (2018) and Le Bihan, he seeks to establish that current approaches to QG do not feature a conceptual gap problem worthy of the analogy to the hard problem of consciousness, and that, if they did, functionalism as such could not dissolve it. Linnemann's analysis seconds Esfeld's expectation that the conceptual problems of the emergence of spacetime will eventually evaporate by working out that none of the current approaches lacks a codification of diachronicity in its structure-which, as he argues, would be the only clear sign that there is no fundamental spacetime structure in QG in the first place.

While accepting the problem of spacetime emergence and its resolution via spacetime functionalism, Baron points out that the original issue regarding empirical coherence in fact also involves a problem concerning the emergence of 'entity', i.e., matter or physical stuff: it is not only important to learn how a notion of beable location arises out of putatively non-spatiotemporal structure but also how the notion of 
a beable itself emerges. According to Baron, the entity problem might, however, just as well be satisfactorily resolved in a functionalist fashion; at least in the context of a matter-enhanced version of loop quantum gravity (LQG), the matter model of quantum field theory (QFT) can plausibly be seen as functionally realised (namelyand just like in the case of spacetime-by LQG's spin-network structure). In order to establish his point, Baron makes use of proposals in the LQG community on how the so-called helon model of particles may explain the emergence of entities with charge from modified versions of LQG's fundamental spin-network structure.

In order to deal with the spacetime functionalism in QG, Rasmus Jaksland-like other contributors - refers to the philosophy of mind. He reads the multiple realisability of the Einstein field equation by different underlying theories postulating different microscopic degree of freedom as a strong case for spacetime functionalism, which is in direct analogy to the central argument in favour of functionalism about mental states. The three theories considered-all of which realise Einstein's field equationare Sakharov's induced gravity (1967), Jacobson's thermodynamic account of gravity (1995), and various forms of entanglement accounts of gravity. Given how naturally these approaches can account for GR, and thus how generic the emergence of Einstein's field equation seems to be, however, the recovery of GR is at best a necessary, but definitely not a sufficient condition for the pursuit-worthiness of an approach to quantum gravity, let alone its correctness.

Finally, Thomas Saad's contribution bridges the gap between space functionalism and philosophy of mind at the level of content rather than just the methodological level: in his essay, he adjudicates whether certain experiences of spatial properties are best accounted for in terms of specific experiential spatial properties (spatial presentationalism) or as functional roles instantiated by suitable spatial properties (spatial functionalism). Saad thus takes functionalism back to the mental. However, in the concrete context he considers, he does not see a clear case for it as being established: whereas the posit of specific experiential spatial properties may lead to the undesirable consequence of spatial properties (currently) not incorporated by physical theories, functionalism about these experiential spatial properties enforces an arguably unacceptable dualism on us - not a path many of us are probably ready to take.

Acknowledgements Open Access funding provided by Projekt DEAL. We acknowledge financial support (for K.C. and N.L.) from the Swiss National Science Foundation (Project 105212 165702) and (for C.W.) from the John Templeton Foundation (Grant 56314, made under a collaborative agreement between the University of Illinois at Chicago and the University of Geneva). The content of this work are solely the responsibility of the authors and do not represent the official views of the Swiss National Science Foundation or the John Templeton Foundation.

Open Access This article is licensed under a Creative Commons Attribution 4.0 International License, which permits use, sharing, adaptation, distribution and reproduction in any medium or format, as long as you give appropriate credit to the original author(s) and the source, provide a link to the Creative Commons licence, and indicate if changes were made. The images or other third party material in this article are included in the article's Creative Commons licence, unless indicated otherwise in a credit line to the material. If material is not included in the article's Creative Commons licence and your intended use is not permitted by statutory regulation or exceeds the permitted use, you will need to obtain permission directly from the copyright holder. To view a copy of this licence, visit http://creativecommons.org/licenses/by/4.0/. 


\section{References}

Albert, D. Z. (2013). Wave function realism. In A. Ney \& D. Z. Albert (Eds.), The wave function: Essays in the metaphysics of quantum mechanics (pp. 52-57). New York: Oxford University Press.

Brown, H. R. (2005). Physical relativity: Space-time structure from a dynamical perspective. Oxford: Oxford University Press.

Brown, H. R., \& Pooley, O. (2006). Minkowski space-time: A glorious non-entity. In D. Dieks (Ed.), The ontology of spacetime (pp. 67-89). Amsterdam: Elsevier.

Hoefer, C. (2000). Energy conservation in GTR. Studies in History and Philosophy of Modern Physics, 31(2), 187-199.

Huggett, N., \& Wüthrich, C. (2013). Emergent spacetime and empirical (in)coherence. Studies in History and Philosophy of Modern Physics, 44(3), 276-285.

Jacobson, T. (1995). Thermodynamics of spacetime: the Einstein equation of state. Physical Review Letters, $75(7), 1260-1263$.

Knox, E. (2013). Effective spacetime geometry. Studies in History and Philosophy of Modern Physics, 44(3), 346-356.

Knox, E. (2014). Spacetime structuralism or spacetime functionalism. Manuscript.

Knox, E. (2019). Physical relativity from a functionalist perspective. Studies in History and Philosophy of Modern Physics, 67, 118-124.

Lam, V. (2011). Gravitational and nongravitational energy: The need for background structures. Philosophy of Science, 78(5), 1012-1024.

Lam, V., \& Esfeld, M. (2013). A dilemma for the emergence of spacetime in canonical quantum gravity. Studies in History and Philosophy of Modern Physics, 44, 286-293.

Lam, V., \& Wüthrich, C. (2018). Spacetime is as spacetime does. Studies in History and Philosophy of Modern Physics, 64, 39-51.

Levin, J. (2018). Functionalism. The Stanford Encyclopedia of Philosophy. https://plato.stanford.edu/ archives/fall2018/entries/functionalism/.

Read, J. (2020a). Functional gravitational energy. British Journal for the Philosophy of Science, 71(1), 205-232.

Read, J. (2020b). Explanation, geometry, and conspiracy in relativity theory. In C. Beisbart, T. Sauer, \& C. Wüthrich (Eds.), Thinking About Spacetime. Birkhäuser: Basel. (forthcoming).

Sakharov, A. D. (1967). Vacuum quantum fluctuations in curved space and the theory of gravitation. Doklady Akademii Nauk SSSR, 177, 70-71.

Wallace, D. (2012). The emergent multiverse: Quantum theory according to the Everett interpretation. Oxford: Oxford University Press.

Publisher's Note Springer Nature remains neutral with regard to jurisdictional claims in published maps and institutional affiliations. 